\title{
RECTAL NARCOSIS IN OPHTHALMIC SURGERY
}

BY

\author{
O. Gayer Morgan and J. M. Lees
}

LONDON

THE question of anaesthesia in ophthalmic surgery is of extreme importance, and although in the majority of operations a local anaesthetic is the one of choice, there is a large proportion of cases in which some other anaesthetic is unavoidable.

For some cases one wishes to find a drug which will cause analgesia or anaesthesia, while at the same time the co-operation of the patient is retained; in others a more complete anaesthesia with a negligible risk of toxic effects or subsequent complications. With the present available material none gives complete satisfaction in all cases. In nervous individuals where a local anaesthetic is required, the large doses of morphia necessary are a frequent source of post-operative nausea or vomiting. In children where the operation is often prolonged, the anaesthetic is difficult to give unless the administrator is highly skilled in the use of the Shipway or intratracheal methods, and the mask and administration in other cases worries the operator and tends to make him hurry over his work. In the elderly where the presence of sepsis or inflammation demands complete narcosis, chronic bronchitis and emphysema are frequently present, and give rise to post-anaesthetic pulmonary complications. Chloroform is probably the most satisfactory, but it is statistically the most ill favoured. Ether is safer, but the apparatus needs more space, and the excessively deep breathing and congestion of the tissues aggravate the difficulties of the operator.

It was, therefore, when looking for a better method that we tried the use of rectal narcosis with avertin. Within certain limits it does wholly fulfil all the requirements demanded for its purpose. In minimal dosage it allays fear and renders tolerable a certain amount of pain without the loss by the operator of the co-operation of the patient. In larger doses it either suffices with a local anaesthetic for operations hitherto requiring general anaesthesia, or by reason of its narcotic effect reduces the amount of anaesthetic required to a minimum. It is simple to give and requires no elaborate apparatus, only a little care in preparation. It has no after-effects at all, the patient having universally constant total amnesia for the operation, in spite of some degree of apparent consciousness, and waking later after a refreshing sleep. It reduces the degree of psychic shock to a minimum. 
Avertin is tribromethyl alcohol, which forms with distilled water a relatively stable solution of about three per cent. at a temperature about $35^{\circ} / 40^{\circ} \mathrm{C}$. If it be heated beyond such a point it decomposes, hydrobromic acid is split off and dibromacetaldehyde is produced. It is supplied either as a powder or as used by us dissolved in amylene hydrate.

Dosage.-The dosage we found to be a matter of a certain amount of experience in order to obtain a uniform and maximum benefit from the narcotic effect. It is calculated at the rate of grams per kilo. of body weight, and in practice this worked out at between 0.06 and 0.09 grams per kilo. As might be expected the highly excitable, or the bull-necked individual required a larger dose than the phlegmatic or spare subject.

The relative effects of the different doses may be considered as below :-

0.06. A minimum basal effect. All co-ordinated movements are retained to a greater or lesser degree. Ocular movements can be elicited by persistent command. The removal from the ward for operation, the preliminary washing, and instillation of cocaine create no reaction, and seem to be regarded in much the same light as the movements and commands of one's barber. Conversation may occur with quite logical trains of thought and expressions of desire to assist the surgeon.

$0 \cdot 07 / 0^{\circ} 08$. These doses are taken together as it is here that temperamental differences, relatively high body weight, height ratios, sex, and age, all have a fairly profound effect upon the degree of narcosis obtained. As a rule the patient is brought to the table quite anaesthetized, some palpable movements may occur on the application of cold stimuli, such as the washout or cocaine drops, but the patient soon settles down again. In operations not involving any stimulation of areas other than those anaesthetized by the cocaine, no further reaction may occur; where, as in enucleation, further areas are stimulated, movements and even low cries of apparent pain may be produced, which are, however, readily controlled, and in no case does anything but a complete state of amnesia for the whole operation exist upon a return of consciousness.

0.09 . This was used as our upper limit on nearly all occasions upon fairly fit patients where a considerable amount of sensory stimulation might be expected. It was very successful in operations such as the extirpation of lacrimal sacs, and plastic operations around the eye, enucleations, etc.

Here, although complete narcosis was never sought or indeed obtained, yet in the majority of cases no added local anaesthetic was used, and yet no external sign of pain or other reaction was visible. 
On two occasions small quantities of $\mathrm{C}$. and $\mathrm{E}$. were used in addition, not so much for the actual need for anaesthesia, but because of our inexperience of the amnesia and lack of shock following operation in the apparently conscious. In one case of a Norwegian leper a dose of 0.075 grams per kilo had been given, and an iridectomy performed upon one eye, the patient lying apparently anaesthetized throughout. His other eye was to be excised, being blind and painful. A preliminary washout had taken place, when the patient suddenly produced the handkerchief which he had been holding in his hand, blew his nose, and asked if he was doing everything to help. He was duly given $\mathrm{C}$. and $\mathrm{E}$. and the excision proceeded with. Later investigation showed that he remembered nothing of the incident, or indeed of anything after the introduction of the rectal tube.

As regards the other forms of anaesthesia combined with avertin, one of us (J.M.L.) has had satisfactory experience of the use of gas and oxygen by the intratracheal route on avertinized patients.

Administration.-The procedure followed throughout has been that suggested by Messrs. Bayer in their clearly expressed handbook. As a routine matter the patient is given a bowel washout by enema at an early hour in the morning, though in cases of emergency this has been omitted with no lack of success.

One hour before the scheduled time of the operation $0.25 \mathrm{gr}$. of morphia with $0.01 \mathrm{gr}$. of atropine are given. (The morphia can be reduced to $0 \cdot 125 \mathrm{gr}$. or omitted altogether. Reference: Young, Lancet, May, 1930).

Half an hour before the operation the calculated amount of avertin is given by rectal tube, at a temperature not exceeding $40^{\circ} \mathrm{C}$. This adherence to temperature is further enforced by the testing of the solution of the avertin by the addition of 2 drops of 1 in 1,000 congo red solution to 5 c.c. of the avertin immediately before use. If any decomposition has occurred, a blue colouration will take place, and any use of the solution in this condition will undoubtedly lead to marked colitis.

The administration of the avertin should take some five or ten minutes, but it is to be noted that the absorption of the drug is so rapid that no extra dose can be given in the event of a return of the first dose by the patient. In such a case the use of avertin must stop for at least four hours.

After the administration the patient falls into a deep sleep and this is greatly hastened by the exclusion of all extraneous noise. Though most of our cases were started in the open ward after merely screening and plugging the ears with wet cotton wool, sleep usually occurred in about ten minutes from the starting of the 
administration. The requisite degree of narcosis does not take place for $25-30$ minutes.

Operation.-The usual period of narcosis in patients who received 0.075 and upwards as a dose, is about $1 \frac{1}{2}-2$ hours. The colour of the skin is often a little cyanosed, and care must be taken to see that the jaw is kept forward all the time to prevent any danger of asphyxia. The corneal reflex is lost early, but the pupil remains small.

The commencement of any additional anaesthesia is exceptionally smooth, and can be kept within fine limits.

Post-operative events. - The patients awake after a period of about 2-3 hours to fall again almost at once into a deep and natural sleep in which they are quite analgesic, and this obviates any need for giving the common opium derivatives. When they awake after this sleep they show an absolute and complete amnesia for all the events subsequent to the giving of the avertin. This has happened in every case, and is one of the most valuable features of the drug.

Secondary effects.-Cyanosis. - It is an undoubted fact that the respiratory centre is affected by use of avertin. In the normal way the respirations are shallow from the start, while the rate of respiration is a little increased. Where an additional anaesthetic is required, if chloroform be used instead of ether or nitrous oxide gas, then the resulting depth of cyanosis is rather startling, although no other signs of anoxaemia appear other than the plum colour of the exposed areas of the skin. These observations may be misleading as all our patients had had morphia, a drug whose depressing action on the respiration and circulation is well known; and some observers have noted that if this be omitted, the cyanosis is not noticeable, and no symptoms of depression occur. Although there is no specific antidote to an overdose of avertin, and a death under these circumstances from respiratory failure is almost inevitable, $\mathrm{CO}_{2}$ is suggested as a stimulant in those cases where one is at all doubtful as to the degree of depth of narcosis obtained, and as being superior to such as camphor, or strychnine, though drugs which have the effect of raising the blood pressure may be of some slight use.

Fall of blood pressure.-Much is made in the literature of the danger of the fall of blood pressure after the administration of avertin. Whilst there is an average fall of $20-35 \mathrm{~mm}$. $\mathrm{Hg}$ before and after operation, yet we are convinced that it is of little importance as a whole.

In cases where blood pressure was taken on admission and several days before the use of avertin, we found that the actual fall was only one of $10-20 \mathrm{~mm}$. $\mathrm{Hg}$, if as much. The expectation of operation caused a pre-operative rise of an additional amount, and 
this is responsible for the apparently very considerable fall under avertin. The pulse was never altered to any degree even after manipulation had taken place under local anaesthetic which had been sufficient to cause movements or outcry.

Venous congestion.-In cases of extirpation of the lacrimal sac, it was found that venous congestion did undoubtedly cause some very slight difficulty, in that oozing tended to make it necessary for an assistant to be very faithful in mopping the blood away, and that a slight increase in the clotting time did occur. In enucleation of the eye it was not found that any additional bleeding took place, or that anything beyond pressure was required to control this.

Colitis-In our series of cases no attack of colitis occurred. It is suggested that this is always directly due to some decomposition of the avertin, and could have been avoided.

The ward sister has always conducted the congo red test herself, as well as superintending the administration of the avertin, and in no case has anything untoward happened. The presence of haemorrhoids causes no extra difficulty, though perhaps it were well to reiterate that under no cirucmstances should the dose of avertin be repeated, because it is thought that all, or nearly all the fluid has been returned.

Contra-indications. - The main contra-indications to the use of avertin have been the presence of any serious degree of nephritis as indicated either by the quantity of albumen in the urine, or by any previous history, and any severe disturbances of the liver.

Age.-We have never used it on patients younger than 16 years of age, but on the other hand the upper limit has been 87 years, the majority being over 65 years.

Sir Francis Shipway, however, quotes a series of severe operations in children which have been eminently successful. He has given doses up to $0.1 \mathrm{gr}$. in children of $5-6$ years of age without seeing any symptom which caused the least anxiety.

(Reference : Brit. Jl. of Anaesthesia. April, 1930).

Sepsis.-In cases of severe toxaemia and in septic cases where one would necessarily be worried as to the result of giving a general anaesthetic, avertin has never caused the least anxiety from the point of view of increasing the toxicity of the patient.

Types of cases.-Altogether more than 50 cases have been dealt with under avertin anaesthesia including cataract extraction, acute glaucoma, excision, evisceration, acute dacryocystitis, removal of the lacrimal sac in chronic dacryocystitis, and some cases of minor and major plastic operations on the eye lids.

In cataract extraction the anaesthesia was perfectly satisfactory and the long sleep and slow recovery afterwards, were of material 
benefit. The dose given was 0.07 or 0.08 and consequently a stitch in the superior rectus muscle was used to depress the globe.

In highly nervous patients this is an ideal method, but in our opinion no artificial fixation of the globe is so satisfactory as the continuous control by the patient when he looks well down towards his feet.

The cases of acute glaucoma were very successful. This operation is usually made much more difficult by the added congestion of the ether anaesthesia. With avertin anaesthesia the patient is quiet, respirations shallow, and the eye in the best possible condition for a successful section, and iridectomy.

In conclusion we consider that avertin anaesthesia well merits a trial in ophthalmic surgery. Naturally, before using it, a thorough familiarity with the method of administration given in the Bayer hand-book and in the other communications on the subject by anaesthetists who have tried it extensively is necessary, but from our experience and that of others, we can conclude that it is not a dangerous drug when given with care, and we think that most of the contra-indications and difficulties have been mentioned in this paper.

\section{A CASE OF MIKULICZ' DISEASE \\ BY \\ H. B. OWen ANd R. S. F. Hennessey \\ UGANDA}

AN African, aged between 50 and 60 years, was admitted to Mulago Hospital, Kampala, Uganda, on February 10, 1930.

The accompanying photographs give a good idea of the appearance presented by the patient on admission. There were enormous swellings in both orbits, and bulging of the lids, greater above than below. On the right side was a firm movable mass, occupying the upper region of the orbit in front of the eyeball, evidently a greatly enlarged lacrymal gland. Below this was a similar smaller growth springing from the palpebral conjunctiva. Occupying the lower region of the orbit was a mass of moderate size originating in the palpebral conjunctiva of the lower lid. On the left side was a similar condition, but the lacrymal gland showed greater enlargement, and the palpebral conjunctiva of the upper lid was the seat of a much more profuse growth with oedema of overlying skin and ulceration of the conjunctival portion of the lid margin. In the lower region of the orbit was a comparatively small mass again taking its origin 